\title{
Necrotizing fasciitis: are tagged-WBC scans underutilized?
}

\author{
McClure $W^{1}$, Ballas $E^{1}$, Kaza ${ }^{1}$, Quon $A^{2}$, Iagaru $A^{3}$ and Douglas $D^{3 *}$ \\ ${ }^{1}$ Department of Radiology, David Grant Medical Center, Travis AFB, CA, USA \\ ${ }^{2}$ Department of Nuclear Medicine, University of California, Los Angeles, California, USA \\ ${ }^{3}$ Department of Radiology, Division of Nuclear Medicine, Stanford University, Stanford, California, USA
}

\begin{abstract}
Necrotizing fasciitis is a rapidly progressing infection of the fascia deep to the skin and superficial to the muscles. This disease is associated with significant morbidity and mortality thus requiring prompt recognition and diagnosis. In some cases, diagnosis of necrotizing fasciitis can be made by physical exam findings alone; however, imaging modalities such as ultrasound, radiographs, computed tomography and magnetic resonance imaging can aid in making the diagnosis. In this case, the findings on computed tomography (CT) and magnetic resonance imaging (MRI) were nonspecific. However, a tagged white blood cell scan, a nuclear medicine study, assisted in the location of an infection source in the patient, leading to a diagnosis of necrotizing fasciitis.
\end{abstract}

\section{Introduction}

The workup of fever of unknown origin can be broad and all encompassing. Clinicians have many tools to utilize to rule in or out various pathologies. An infrequently used imaging modality, Indium-111 white blood cell (In-111 WBC) imaging is a nuclear medicine procedure that can provide valuable information about the diagnosis of infections. The scan entails removing white blood cells from the patient, tagging them with Indium-111 radioisotope, and then injecting them back into the patient intravenously. The tagged cells will localize to the source of infection, which can be seen on subsequent scanning. In-111 WBC scan has been shown to aid in the diagnosis of infection, particularly osteomyelitis and vascular graft infections, as well as in soft tissue sarcoma and neoplasm [1-5]. This case report details the use of In-111 WBC scan to diagnose necrotizing fasciitis, which is historically not a typical use of In-111 WBC scan.

\section{Case report}

A 46-year-old man with a history of IV drug use, heart failure and renal failure one-month status post heart and kidney transplant presented with fevers, septic shock and severe right lower extremity pain. Workup of this patient's condition included computed tomography (CT) and magnetic resonance imaging (MRI) scans, both demonstrating non-specific right lower extremity edema. The finding of edema is nonspecific and can be seen with many processes including fluid overload. Subcutaneous emphysema is a specific finding for necrotizing fasciitis; however, this finding was not seen in this case. In fact, subcutaneous gas is seen only in the minority of cases [6]. A tagged-WBC scan was ordered for workup of fever of unknown origin and revealed marked increased WBC accumulation in the right lower extremity with a linear pattern confined to the fascial planes (Figure 1). The patient was taken to surgery and necrotizing fasciitis due to Klebsiella pneumonia was confirmed via pathology and histology (Figure 2).

\section{Discussion}

Necrotizing fasciitis is a surgical emergency with significant morbidity and mortality, characterized as a rapidly progressing
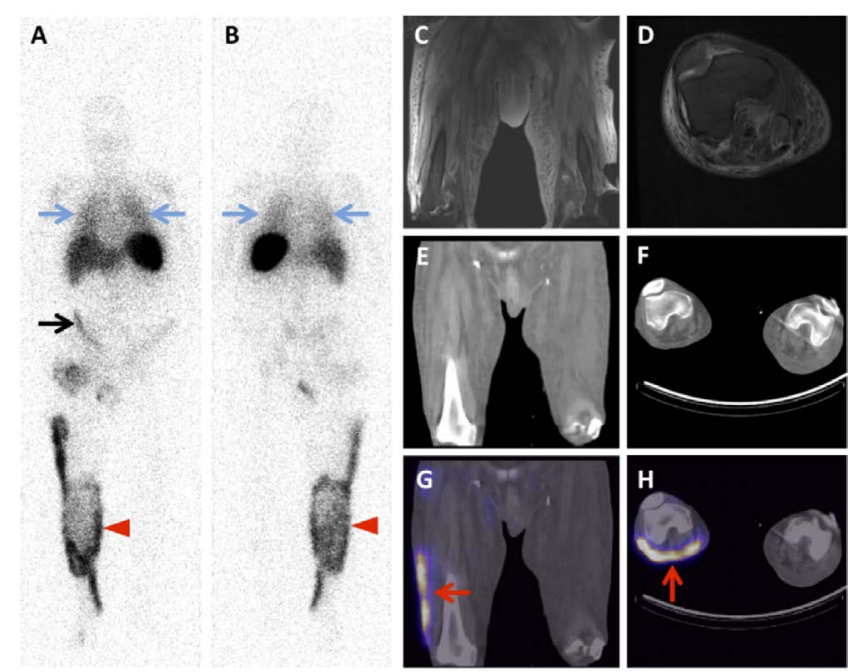

Figure 1. (A) Anterior and (B) posterior planar images from an In-111 WBC scan showed marked increased WBC accumulation in the right leg (red arrowheads). Additional findings included diffuse uptake in the lungs (blue arrows) reflecting pneumonia and linear uptake in the right lower quadrant of the abdomen (black arrow) reflecting an infected renal transplant incision. MRI (T2 fat saturated) sequences of the bilateral thighs (C) and right knee (D) demonstrate bilateral T2 hyperintensity in the subcutaneous tissues reflecting edema. CT images of the bilateral thighs (E) and bilateral knees (F) demonstrate increased density in the subcutaneous tissues reflecting edema. The SPECT-CT images of the bilateral thighs (G) and knees $\mathbf{( H )}$ demonstrate WBC accumulation in a linear pattern that localizes to the fascial plane between the skin and the musculature

infection of the fascia deep to the skin and superficial to the muscles [7]. This infection is typically polymicrobial resulting from organisms such as Clostridium, Proteus, E. coli, Bacteroides, Enterobacteriaceae,

${ }^{\star}$ Correspondence to: David Douglas,Department of Radiology, Division of Nuclear Medicine, Stanford University, Stanford, California, USA. E-mail: ddouglas@stanford.edu

Key words: necrotizing fasciitis, wbc scan, klebsiella, infection

Received: August 13, 2018; Accepted: August 20, 2018; Published: August 24, 2018 

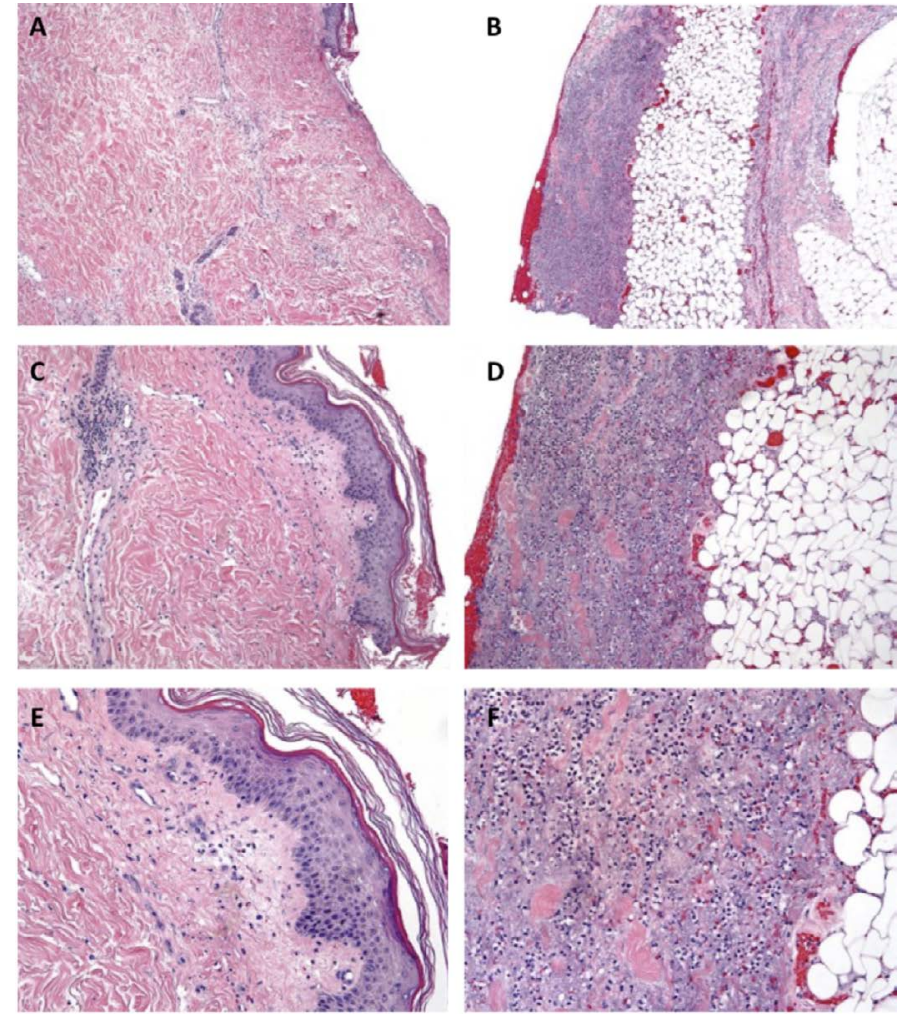

Figure 2. Epidermis and dermis 4x (A) 10x (B) 20x (C): Denuding epidermis and underlying dermis with interstitial and perivascular mixed inflammatory infiltrate composed of neutrophils and lymphocytes with necrotic adnexal structures. Deep dermis and subcutis 4x (D) 10x (E) 20x (F): Prominent mixed lymphocytic and neutrophilic inflammatory infiltrate in the deep dermis and subcutis

Klebsiella or Group A strep [8-11]. Rapid diagnosis is key in management of this disease as treatment includes prompt surgical debridement and IV antibiotics.

Currently, the imaging modality of choice to evaluate for necrotizing fasciitis is MRI, with a sensitivity of $93 \%$. CT is also used but has a lower sensitivity of $80 \%$ [12]. Necrotizing fasciitis is rarely diagnosed with a nuclear medicine scan. To our knowledge, there is only one case reported on a Tc-99m labeled WBC scan in 1999[13] and one case reported on a Ga-67 scan in 2003 [14]. Necrotizing fasciitis has not been reported on an In-111 WBC scan. Our In-111 labeled WBC scan and the two other nuclear medicine scans of necrotizing fasciitis in the literature demonstrate a strikingly similar linear pattern of uptake on the planar imaging. As a result of these similarities and findings, we feel that this case brings up a couple of important questions. Does a WBC scan potentially have a higher sensitivity and/or specificity for detection of necrotizing fasciitis than CT and/or MRI? Could a nuclear medicine WBC scan be beneficial for surgical planning? In-111 WBC scans have historically been used to aid in the workup of fevers of unknown origin, and to diagnose infections, particularly osteomyelitis and vascular graft infections. Lewis et al. reported that In-111 WBC scans were useful less than half the time at their care center; however, they suggest that targeted use of the study to evaluate for specific infections could be considered. Our case report suggests further exploration about the utility of the In-111 WBC scan, as it may be underutilized as a tool in diagnosing and surgical planning in patients with necrotizing fasciitis.

\section{References}

1. Lewis SS, Cox GM, Stout JE (2014) Clinical utility of indium 111-labeled white blood cell scintigraphy for evaluation of suspected infection. Open Forum Infect Dis 1: ofu089. [Crossref]

2. Lamki LM, Kasi LP, Haynie TP (1988) Localization of indium-111 leukocytes in noninfected neoplasms. J Nucl Med 29: 1921-1926. [Crossref]

3. Palestro CJ, Love C, Tronco GG, Tomas MB, Rini JN (2006) Combined labeled leukocyte and technetium $99 \mathrm{~m}$ sulfur colloid bone marrow imaging for diagnosing musculoskeletal infection. Radiographics 26:859-870.

4. Schmidt KG, Rasmussen JW, Wedebye IM, Frederiksen PB, Pedersen NT (1988) Accumulation of indium-111-labeled granulocytes in malignant tumors. $J \mathrm{Nucl}$ Med 29: 479-484. [Crossref]

5. Fong V, Johnson L (2017) Indium-111 labeled leukocyte accumulation in extremity soft tissue sarcoma. Radiol Case Rep 12:383-385.

6. Wang JM, Lim HK (2014) Necrotizing fasciitis: eight-year experience and literature review. Braz J Infect Dis 18: 137-143. [Crossref]

7. Li B, Zhao Y, Liu C, Chen Z, Zhou D (2014) Molecular pathogenesis of Klebsiella pneumoniae. Future Microbiol 9:1071-1081.

8. Monie M, Drieux L, Nzili B, Dicko M, Goursot C, et al. (2014) Klebsiella pneumoniae necrotizing fasciitis of the leg in an elderly French woman. Clin Interv Aging 9:11711174.

9. Fugitt JB, Puckett ML, Quigley MM, Kerr SM (2004) Necrotizing fasciitis. Radiographics 24: 1472-1476. [Crossref]

10. Rehman J, Kaynan A, Samadi D, Fleischmann J (1999) Air on radiography of perinea necrotizing fasciitis indicates testis involvement. J Urol 162: 2101. [Crossref]

11. Lee BE, Robinson JL (1999) The use of technetium-99m-labeled white blood cell scan in the management of a case of group A streptococcus necrotizing fasciitis with polymyositis. Clin Infect Dis 28: 153-154. [Crossref]

12. Tso DK, Singh AK (2018) Necrotizing fasciitis of the lower extremity: imaging pearls and pitfalls. Br J Radiol. 91: 20180093. [Crossref]

13. Callahan EF, Adal KA, Tomecki KJ (2000) Cutaneous (non-HIV) infections. Dermatol Clin 18: 497-508, x. [Crossref]

14. Hakkarainen TW, Kopari NM, Pham TN, Evans HL (2014) Necrotizing soft tissue infections: review and current concepts in treatment, systems of care, and outcomes. Curr Probl Surg 51: 344-362. [Crossref]

Copyright: (C2018 McClure W. This is an open-access article distributed under the terms of the Creative Commons Attribution License, which permits unrestricted use, distribution, and reproduction in any medium, provided the original author and source are credited. 